\title{
WORKPLACE WELLNESS USING ONLINE LEARNING TOOLS IN A HEALTHCARE SETTING
}

\section{Holly Blake and Emily Gartshore}

\section{Abstract}

The aim was to develop and evaluate an online learning tool for use with UK healthcare employees, healthcare educators and healthcare students, to increase knowledge of workplace wellness as an important public health issue. A 'Workplace Wellness' e-learning tool was developed and peer-reviewed by 14 topic experts. This focused on six key areas relating to workplace wellness: work-related stress, musculoskeletal disorders, diet and nutrition, physical activity, smoking and alcohol consumption. Each key area provided current evidencebased information on causes and consequences, access to UK government reports and national statistics, and guidance on actions that could be taken to improve health within a workplace setting. 188 users $(93.1 \%$ female, age 18-60) completed online knowledge questionnaires before $(n=188)$ and after $(n=88)$ exposure to the online learning tool. Baseline knowledge of workplace wellness was poor ( $n=188$; mean accuracy $47.6 \%$, s.d. 11.94). Knowledge significantly improved from baseline to post-intervention (mean accuracy $=77.5 \%$, s.d. 13.71) $(t(75)=-14.801, p<0.0005)$ with knowledge increases evident for all included topics areas. Usability evaluation showed that participants perceived the tool to be useful $(96.4 \%)$, engaging $(73.8 \%)$ and would recommend it to others (86.9\%). Healthcare professionals, healthcare educators and pre-registered healthcare students held positive attitudes towards online learning, indicating scope for development of further online packages relating to other important health parameters.

\section{INTRODUCTION AND BACKGROUND}

Poor public health and wellbeing is globally accepted as a significant burden to healthcare services, due to the rising incidence of morbidity and mortality related to preventable chronic conditions (World Health Organisation (WHO), 2014; Department of Health (DH), 2014; Centre for Disease Control and Prevention, 2012). As such, there is an international call for population behaviour change to reduce smoking, reduce alcohol consumption, improve diet and increase physical activity (Public Health England, 2014; WHO, 2014; Office for National Statistics (ONS), 2013; DH, 2012c, 2011a, 2010; Mulgan, 2010). 
Creating a 'culture of health' has been advocated to promote health behaviour change and improve the health of individuals (Shareck, Frohlich, and Poland, 2013; Black and Frost, 2011; WHO, 2010b; Musich, Schubiner and McDonald, 2009; Black, 2008). This 'culture of health' can be achieved through settings-based health promotion (WHO, 2011). Workplaces are an integral setting for improving public health (Black, 2008; Black and Frost, 2011), and workplace health promotion interventions have been shown to reduce the burden of illness to both employees and the employer (Musich et al, 2009; Black, 2008; Black and Frost, 2011; WHO, 2010b; Department of Work and Pensions, 2011, 2013).

The UK National Health Service (NHS) is one of the world's largest publicly funded health services, estimated to be the fifth largest employer worldwide, with approximately 1.7 million employees working across England, Wales, Scotland and Northern Ireland. For healthcare employees and healthcare students (Blake and Harrison, 2013; Mooney et al, 2011), advocating workplace wellness is particularly salient from two perspectives. Firstly, many employees working within the UK NHS have been shown to have poor health profiles (Blake et al, 2011) and the National Health Service (NHS) workforce holds higher sickness absence rates than other public sector and private sector organisations (Chartered Institute of Personnel and Development (CIPD), 2014; ONS, 2014; Boorman, 2009). The health and wellbeing of healthcare employees is vital for improving organisational engagement (Point of Care Foundation, 2014; West et al, 2011), confidence to promote healthy lifestyle behaviours to patients (Blake and Harrison, 2013), patient experience and patient outcomes (DH, 2011c; West et al, 2011; Boorman, 2009). As such, healthcare employees are important recipients of workplace health promotion. Indeed, the Five Year Forward Plan proposed by NHS England advocates that a radical upgrade in prevention and public health is required for the sustainability of the NHS, as well as economic prosperity of the country. This plan supports new workplace incentives to promote employee health and wellbeing. Secondly, since workplace wellness is an important element of public health promotion within the UK, NHS employees (and healthcare students as the 'next generation' of NHS employees) should have a clear understanding of this subject area, in order that they are empowered to promote health and wellbeing amongst their peers, students, patients and the general public (Blake and Harrison, 2013). Nevertheless, knowledge about healthy lifestyle behaviours appears to be inadequate in NHS employees (Mo, Blake and Batt, 2011). 
Educational intervention is therefore appropriate in order to increase understanding about important health behaviours (manifestations, causes and consequences), and how employees and organisations can make efforts to improve and support healthy lifestyle choices in the NHS workplace setting.

Technology has been nationally recognised within the UK for its potential to improve health by transforming the quality of health and care services, whilst reducing costs (DH, 2012a, 2012b; Government Digital Strategy, 2013; Care Act, 2014; NHS, 2014; National Information Board (NIB), 2014). The NHS Five Year Plan sets out a national drive to embed and enhance information technology within health and care services. Making it vital for all members of the UK healthcare workforce to have the knowledge and skills necessary to fulfill their roles in the use of information, data and technology (NIB, 2014).

Moreover, technology has a key part in the delivery of healthcare education worldwide, with the use of elearning and blended learning approaches found to enhance knowledge, skills and learner satisfaction (Back et al, 2014; Barnard-Ashton, Koch and Rothberg, 2014; Lotrecchiano et al, 2013; Smyth et al, 2012, Ward, Moule and Lockyer, 2009). Education delivered through online tools allow for a wide footprint, potentially providing a low-cost, easily accessible means of health education for large numbers of healthcare staff and students (Blake, 2010; NIB, 2014; DH, 2011b). Online tools have shown to be an effective method for increasing knowledge in a variety of subject areas, whilst also providing flexibility and accessibility (Back et al, 2014; Keefe and Wharrad, 2012; Wharrad et al, 2012; Windle et al, 2010).

\section{AIM}

To develop an online learning tool about workplace wellness and evaluate its usefulness and acceptability for enhancing knowledge in individuals working in a UK healthcare setting. These include: [1] healthcare employees (working within the UK NHS); [2] healthcare educators (teaching healthcare students based within UK NHS hospitals); and [3] healthcare students (based within UK NHS hospitals and next generation of healthcare employees).

\section{DESIGN AND DEVELOPMENT}

In the development phase, a 60-minute workplace wellness e-learning tool was created using Articulate Presenter (2013). Development adhered to the Centre for Excellence in Teaching and Learning in Reusable 
Learning Objects (RLO-CETL) Agile Development Workflow (2009) (Figure 1). The process involved development of a 'storyboard' containing the draft content, resources and details of interactive elements, followed by development of draft content into an online resource, with an iterative process of peer review at each stage.

[insert Figure 1 here].

Content included key topic areas of work-related stress (Health and Safety Executive (HSE), 2010), musculoskeletal disorders (HSE, 2013), diet and nutrition (WHO/WEF, 2008), physical activity (NICE, 2008), smoking and alcohol consumption (WHO/WEF, 2008). Each key area provided current evidence-based information on causes and consequences, access to UK government reports and national statistics, and guidance on actions that could be taken to improve health within a workplace setting. Presentation of materials included the use of images, interactions, quizzes and activities (Joint Information Systems Committee, 2004) since interactivity is known to enhance engagement of the user (Cheong Li, Wong and Cheung, 2015; Clark and Mayer, 2011; Horton, 2006; Cook, Levinson and Garside, 2008, 2010)

Initial content was drafted by a nurse and a health psychologist; this was reviewed by a panel of 14 UK-based workplace wellness experts with academic and practice expertise, who were identified through national workplace health networks and publications and commented on the accuracy and appropriateness of design and content (RLO-CETL, 2005). Once content was finalised, the online resource was developed and further peer reviewed by the panel of experts. The overall content review process took 8 weeks and responses were provided by the expert panel electronically. The final online tool was pilot tested with a group of 14 healthcare staff and students. Pilot participants were sent a link to the tool by email, and asked to complete the final version of the tool in their own time, record the time to complete, and raise any potential problems with future use; feedback was provided by return email. This user feedback allowed for the online tool to be developed to meet the learning needs of the group and improve essential elements such as the use of activities and images, allowing users to attain a higher level of understanding (Horton, 2006). Pilot testing resulted in minor amendments, including revisions to layout, formatting, and correction of minor technical issues. The resulting online learning tool is available here: 
http;//www.nottingham.ac.uk/nmp/sonnet/lifestyle/workplace-wellness/presentation.html (Blake and Gartshore, 2014a; Gartshore and Blake, 2014b).

\section{RESEARCH DESIGN}

\section{Evaluation procedure}

Ethical approval was granted by the local institutional Research Ethics Committee. A one-group pre and posttest method was used to examine changes in knowledge following exposure to the workplace wellness educational intervention. This approach was selected since evaluations based only on subjective user opinions have been criticised for not fully reflecting educational outcomes (Holloway and Wheeler, 2013; Bloomfield, 2008).

\section{Sample}

The online learning tool was made accessible to a total of 2689 individuals. These were comprised of: 2390 healthcare students (2050 Nursing students, 194 Midwifery students and 146 Physiotherapy students), 263 university healthcare educators, and 36 healthcare employees based at a single NHS hospital trust. All these individuals were sent an email with the link to the online tool, with an invitation to complete the package. The email invitation was sent from a university Work, Health and Wellbeing champion. It included a paragraph with brief information about the importance of health and wellbeing for employees and students based within healthcare settings, and highlighted the national drive to improve health and wellbeing within the NHS. No incentives were offered for completion of the tool, and it was offered as optional professional development training. To our knowledge, at the time of the study, there was no comparable, evidence-based online learning tool available for employees or students working within a healthcare setting (either in the UK or internationally), and no tools previously developed with specific relevance to UK healthcare settings that had received input and review from experts and users within this target population. Of those who received the tool, 188 participants opted to complete the package during a seven-week period from November 2013 January 2014.

\section{Data collection}


Data were collected from those who completed the tool using a multiple-choice online questionnaire designed to assess participant's knowledge before and after completion of the workplace wellness e-learning tool. The questionnaire was embedded within the learning tool, and consisted of 20 knowledge questions which were derived from the online learning content by two developers, using concepts from the constructive alignment theory (Biggs, 1999). These were close-ended questions providing presubscribed response alternatives, including for example, recommended levels for health behaviours, healthy choices, or national statistics around the included topic areas. For quality control, the questions were validated by the panel of workplace wellness experts prior to use. Knowledge level of individuals was deemed to be low if accuracy of response was below $50 \%$ overall. To assess the effectiveness, usability and the context of learning, the postquestionnaire included seven items from a nationally developed evaluation tool for online learning tools (RLOCETL, 2005), using recommended questions to complement the development workflow used during this project (RLO-CETL, 2009). The questions assessed participant perceptions of the usability of the online learning tool, including perceptions of the usefulness of the tool for learning about workplace wellness and whether they found it engaging, details of where participants accessed the tool, participant's perceptions of their own level of computer competence, and whether participants would recommend the tool to others.

\section{Data Analysis}

Knowledge items were marked and standardised scores were derived by the researcher and cross-checked by an independent external marker. Data were analysed using Statistical Package for the Social Sciences (SPSS) Version 21.0. Analysis included descriptive statistics and paired t-tests for pre-post analysis. Significance was set at $\mathrm{p}<0.05$ for all statistical comparisons.

\section{RESULTS}

\section{Response rate and demographic characteristics}

There were 188 participants who voluntarily opted to complete the learning tool and evaluation measures as professional development training within the study period (a conservative $6.9 \%$ of those invited). The sample was predominantly female $(93.1 \%, n=175)$, age ranged from $18-60$ years $(52.1 \%$ aged from $18-29$ years). Over half of the sample $(53.7 \%, n=101)$ reported that they were employees working within a healthcare setting. Of 
all those accessing the tool, 69.1\% $(n=130)$ of participants were higher education students (these categories are not always mutually exclusive, $22.8 \%$ of our participants were employees at the healthcare institution, but registered for university study in a healthcare discipline concurrently). Characteristics of those who did not opt to complete the professional development package during the study period are unknown.

\section{Pre and post knowledge comparison}

Distribution and normality of data was considered through the use of P-P Plots, normality and skewness tests. At baseline, knowledge scores were normally distributed; post-intervention, the knowledge data showed a normal distribution curve with a negative skew.

Knowledge accuracy scores were calculated for each participant based on their percentage of correct responses to the questionnaire items. Mean response accuracy at pre-questionnaire assessment $(n=188$; $47.60 \% \pm 11.94 \%)$ indicated that knowledge of workplace wellness was highly variable, but on average, relatively poor at the outset (averaging $<50 \%$ accuracy). There were no significant differences in prequestionnaire assessment scores with demographic characteristics (age, gender, employment or educational status). Paired t-test showed a significant increase in accuracy from pre to post questionnaire knowledge scores: $t(75)=-14.801, p<0.0005$. Accuracy increased from $47.60 \%$, s.d. 11.94 at pre-assessment to $77.5 \%$, s.d. $13.71(p<0.0005)$ at post-assessment with a large effect size of 1.7 (Cohen, 1988). Analysis of individual question items showed significant improvements in knowledge for all 20 individual question items indicating improvements in all subject sub-sections. At baseline, the percentage of correct responses for each item ranged from $13.5 \%-89.9 \%$. At post-questionnaire, the percentage of correct responses for each item ranged from $43 \%-100 \%$. At pre-questionnaire assessment, only one item achieved correct responses from over $75 \%$ of participants. By post-questionnaire assessment, there were 14 questions items for which over $75 \%$ of participants gave the correct response. ANOVA testing showed there were no significant differences in prequestionnaire or post-questionnaire assessment scores with demographic characteristics (age, gender, employment or educational status).

\section{User evaluation}


The majority of participants completing the e-learning tool $(n=88)$ accessed the resource at home $(n=62$, 70.5\%) and described themselves as having high or very high computer competence $(n=57,64.8 \%)$, showing the context and ability of users prior to the e-learning tool (RLO-CETL, 2005). Selected usability questions from the RLO-CETL(2005) evaluation toolkit were completed by 84 participants to assess the perceived usability and effectiveness of the e-learning tool. This revealed that the vast majority rated the tool as a useful resource (96.4\%; $n=81), 88.1 \%(n=74)$ enjoyed being able to learn on their own, $73.8 \%(n=62)$ found it engaging and $86.9 \%(n=73)$ would recommend the tool to others. A minority felt that the tool was too long; no other barriers to completion were raised. Comments reflected that users particularly enjoyed the information and content provided, the requirement to actively engage with the tool, the use of interactive quizzes throughout, and the use of engaging images, audio and interactions.

\section{DISCUSSION}

An online educational tool was developed to promote learning about workplace wellness in healthcare employees, healthcare educators and healthcare students in the UK. Evaluation showed low levels of knowledge at the outset, and increases in learning, irrespective of demographic characteristics, occupational or educational status. A significant increase was observed in knowledge about workplace wellness following exposure to the tool, in all subsections including work-related stress, musculoskeletal disorders, diet and nutrition, physical activity, smoking and alcohol consumption. Comparison of test scores before and after exposure to the tool is valuable in assessment of educational outcome since evaluation of e-learning methodology is often criticised for a focus on subjective perceptions and a lack of objective evidence (Bloomfield, 2008). Although this was a one-group design, embedding the pre and post testing within the tool itself ensured that testing took place immediately before and after the intervention, increasing the plausibility that the intervention was the cause of any identified changes (Polit and Tatano-Beck, 2013, 2008).

At the outset, participants had relatively poor knowledge of all subject areas included under the general area of workplace wellness (with less than $50 \%$ accuracy), irrespective of their age, gender, employment or educational status. This was surprising given that our participants include qualified healthcare professionals, healthcare educators and healthcare students many of which have (or aim to have) a responsibility for health 
promotion as part of their role. This study suggests that there is a need to increase knowledge about workrelated stress, musculoskeletal disorders, diet and nutrition, physical activity, smoking and alcohol consumption, and increase understanding about methods of promoting health through the workplace setting. This is important not least for promoting the health and wellbeing of our target population in the workplace setting, but also in ensuring that those with a responsibility for promoting health to patients and the general public are empowered to do so. This is particularly pertinent for healthcare employees of current and future generations; research has indicated there is a pressing need to promote and support healthy lifestyle behaviours in NHS employees and healthcare students (Blake and Chambers, 2011; Blake et al, 2012; Malik et al, 2011; DH 2009). This is because health in NHS employees has been associated with sickness absenteeism rates (alongside job-related and organisational factors) (CIPD, 2009; DH, 2009c), confidence to promote health to patients (Blake and Harrison, 2013) patient satisfaction (Point of Care Foundation, 2014; West et al, 2011) and patient outcomes (DH, 2011c, 2012c; West et al, 2011; Boorman, 2009). This online learning tool acts to reinforce a 'culture of health' (Musich et al, 2009), by signposting healthcare professionals, educators and students to available support and encouraging them to provide health promotion information to others. This study focused on increasing knowledge of workplace wellness in our target populations, and it was beyond the scope of the study to measure objective changes in behaviour or attitudes resulting from the increased knowledge; although attitudes undoubtedly play a role in individual health behaviours, and cultural change within organisations. However, the implications of this learning tool as an intervention are potentially considerable, and this needs to be tested with measurement of change in attitude, health behaviour, and organisational outcomes such as sickness absenteeism and workforce retention.

This study demonstrates that online learning is an appropriate platform for education about workplace wellness in healthcare professionals and students. We observed statistically significant increases in knowledge following exposure to the tool and this was corroborated by participant self-reports that their knowledge and understanding had improved. User evaluation demonstrated that the online learning tool is highly acceptable to participants, and the vast majority of those who completed it perceived the tool to be engaging, a useful mechanism for learning, and would recommended it to others. Respondents completing the tool $(n=88)$ accessed the learning tool from a variety of locations including university $(15.9 \%, n=14)$ and whilst at work $(13.6 \%, n=12)$, with most accessing it (and expressing a preference for accessing it) from their homes (70.5\%, 
$n=62$ ), which highlights the easy access and flexibility of online learning approaches. Computer confidence was high in our sample, which is relevant since poor computer confidence is an identified barrier to online learning (McVeigh, 2009). This may indicate a voluntary response bias, due to the self-selecting nature of the study, which may mean that those who have low computer confidence choose to not participate (Deniz and Citak, 2010). Alternatively, it may simply reflect the requirements of computer competency in the target populations (DH, 2004; Atack and Rankin, 2002). The low completion rate by healthcare students may be related to the fact that the tool was made available by email link to students as optional supplementary learning, the vast majority of whom were out on practice placements during the study period and balancing academic studies with clinical training. Completion by students may be increased by embedding the tool within health promotion sessions within mainstream healthcare education. The vast majority of completers felt positively towards the package as a mechanism for learning, and although a small number of individuals commented on the over-length of the tool, it should be recognised that this method of learning takes less time than classroom teaching (Jeffries, 2001; Feng et al, 2013; Lahti, Hatonen and Valimaki, 2014), whilst being adaptable and flexible to meet the diverse learning needs of users. This tool may therefore be useful as a low cost, mass reach tool to raise awareness about the importance of workplace wellness as a key public health issue within healthcare settings.

Whilst there was a large number $(n=188)$ of employees and students who voluntarily opted to complete the tool in their own time as part of this evaluation, this represented a low proportion of the overall population to whom the online learning was offered. This was not unexpected, since deciding to undertake supplementary learning over and above work commitments or studies is the individual's responsibility, and there is a tendency to select continual professional development (CPD) opportunities which are perceived to be directly relevant to specific job roles and areas of expertise (Ousey and Roberts, 2012). For example, this may have appealed more to those who were particularly interested in health promotion; with this form of self-selection bias one might anticipate higher than average knowledge scores, as participants are likely to be those with an evident interest in the subject (Deniz and Citak; 2010). However, this does not seem to be the case since baseline knowledge of all health topics within the workplace wellness tool was low. Other obstacles to CPD have been raised, such as employers not always being supportive about engagement in CPD training (Hegney et al, 2010). However, it has been recommended that education providers should offer non-traditional study packages in 
the form of online learning, in order to maximise the uptake of continuing professional development (Ousey and Roberts, 2012).

\section{CONCLUSIONS}

This study identifies low levels of knowledge of workplace health and wellbeing amongst our sample of NHS employees, healthcare educators and healthcare students. It demonstrates a need for education and training in this important field of public health, particularly for those working in, or associated with, the National Health Service in the UK, although this is likely to have relevance in healthcare settings elsewhere. An innovative evidence-based online learning tool was developed which included work-related stress, musculoskeletal disorders, diet and nutrition, physical activity, smoking and alcohol consumption, together with guidance on methods of promoting health and making lifestyle changes in the workplace setting. Knowledge about workplace wellness significantly increased following exposure to this interactive tool, which was well-evaluated by users. As such we recommend use of the tool to support workplace wellness promotion and education within UK healthcare workplaces, which may provide a blueprint for the development of comparable tools with relevance to other healthcare environments internationally. Whilst awareness of this subject area has been raised in our UK-based healthcare setting, it is yet to be tested whether increases in knowledge translate into tangible changes in individual health, lifestyle behaviour choices or workplace health promotion practices, which has direct relevance for our future and current public health workforce.

Conflict of interest

The authors declare no conflict of interest. 



\section{References}

Articulate (2013). E-learning software and authoring tools. Available online at: www.articulate.com (Accessed 05.09.2015).

Atack, L. and Rankin, J. (2002). A descriptive study of registered nurses' experiences with web based learning. Journal of Advanced Nursing 40(4): 457-465.

Back, D., Haberstroh, N., Antolic, A., Sostmann, K., Schmindmaier, G. and Hoff, E. (2014) Blended learning approach improves teaching in a problem-based learning environment in orthopedics - a pilot study. BMC Medical Education 14 (17).

Barnard-Ashton, P., Koch, L. and Rothberg, A. (2014) The influence of blended learning on student performance in an undergraduate occupational therapy curriculum. South African Journal of Occupational Therapy 44 (1): 75-80.

Biggs, J. (1999). Teaching for Quality Learning at University. Buckingham: SRHE and Open University Press.

Black, C. (2008) Working for a healthier tomorrow. London: The Stationary Office.

Black, C. And Frost (2011). Health at work - an independent review of sickness absence (Cm8205). London: The Stationary Office.

Blake, H. (2010). Computer-Based Learning Objects in Healthcare: The Student Experience. International Journal of Nursing Education Scholarship 7(1): 1-15.

Blake, H. and Chambers, D. (2011). Supporting nurse health champions: Developing a 'new generation' of health improvement facilitators. Health Education Journal 1-6.

GARTSHORE, E and BLAKE, H, 2014. "Workplace Wellness": online learning package. University of Nottingham. Available at: http://www.nottingham.ac.uk/nmp/sonet/rlos/lifestyle/workplace-wellness/presentation.html. Last accessed 04.07.2016. 
Blake, H. and Gartshore, E. (2014). Development and evaluation of an online learning tool to enhance knowledge of workplace wellness in healthcare disciplines In: Proceedings of the 10th UK Society for Behavioural Medicine Annual Scientific Meeting, 3-4 December, Nottingham.

Blake, H. and Harrison, C, (2013). Health behaviours and attitudes towards being role models. British Journal of Nursing 22 (2): pp.86-94.

Blake, H., Malik, S., Mo, P. and Pisano, C. (2011). 'Do as say, but not as I do': are next generation nurses role models for health? Perspectives in Public Health 131(5): 231-239.

Blake, H., Mo, P., Lee, S. and Batt, M. (2012). Health in the NHS: Lifestyle behaviours of hospital employees. Perspectives in Public Health 132: 213-215.

Bloomfield, J. (2008). Using computer assisted learning for clinical skills education in nursing: integrative review. Journal of Advanced Nursing 63 (3): 222-235.

Boorman, S. (2009). NHS Health and Wellbeing Final Report November 2009. Available online at: www.dh.gov.uk/publications (Accessed: 02.09.2015)

Care Act 2014. (2014) Available online at: http://www.legislation.gov.uk/ukpga/2014/23/contents/enacted. (Accessed: 02.09.2015)

Centre for disease control and prevention (CDC)(2012). Ten leading causes of death and injury; causes of death by age group. Available online at: http://www.cdc.gov/injury (Accessed 02.09.2015).

Centre for Excellence in Teaching and Learning in Reusable Learning Objects (RLO-CETL) (2009) CETL Agile Development Workflow. Available online at: www.rlo-cetl.ac.uk/ (Accessed 04.09.15).

Centre for Excellence in Teaching and Learning in Reusable Learning Objects (RLO-CETL) (2005) Evaluation Toolkit. Version 1. Available online at: www.rlo-cetl.ac.uk (Accessed 04.09.15).

Chartered Institute of Personnel and Development (CIPD) (2014). Absence Management Annual survey report 2014. Available online at: www.cipd.co.uk (Accessed: 02.09.2015) 
Cheong Li, K., Wong, T. and Cheung, S. (2015) Technology in Education: International Conference, ICTE 2014, Hong Kong, China, July 2-4, 2014. Revised Selected Papers (Communications in Computer and Information Science). UK: Springer

Clark, R. and Mayer, R. (2011) e-Learning and the Science of Instruction: Proven Guidelines for Consumers and Designers of Multimedia Learning (Essential Knowledge Resource). New York: John Wiley \& Sons Inc.

Cohen, J. (1988). Statistical power analysis for the behavioural sciences. 2nd ed. Hillsdale: Erlbaum.

Cook, D., Levinson, A., Garside, S., (2008). Internet-based learning in the health professions. Journal of American Medical Association 30(10): 1181-1196.

Cook, D., Levinson, A., Garside, S., (2010). Instructional design variations in internet-based learning for health profession education: a systematic review and meta-analysis. Academic Medicine 85 (5): 909-922.

Deniz, K. and Citak, G. (2010). The investigation of factors affecting university students' attitudes towards participation in scientific research. Procedia Social and Behavioural Sciences 2: 5183-5189.

Department of Health (DH) (2004) The NHS Knowledge and skills framework and the development review process. London: Her Majesty's Stationary Office.

Department of Health (DH) (2010). Healthy Lives, Healthy People: Our strategy for public health in England. CM7985 London: DH.

Department of Health (DH) (2011a). Changing Behaviours, Improving Outcomes: A social marketing strategy for public health. London: The Stationary Office.

Department of Health (DH) (2011b). Framework for Technology Enhanced Learning. Available online at: www.dh.gov.uk/publications (Accessed: 17.09.2015).

Department of Health (DH) (2011c). Healthy Staff, Better Care for Patients Realignment of Occupational Health Services to the NHS in England. Available online at: www.dh.gov.uk/publications (Accessed: 17.09.2015). 
Department of Health (DH) (2012a). Digital Strategy: Leading the Culture Change in Health and Care.

Available online at: www.dh.gov.uk/publications (Accessed: 06.09.2015).

Department of Health (DH) (2012b). Power of Information. Available online at: www.dh.gov.uk/publications (Accessed: 06.09.2015).

Department of Health (DH) (2012c). Improving outcomes and supporting transparency, A public health outcomes framework for England, 2013-2019. Available online at: www.dh.gov.uk/publications (Accessed 02.09.2015)

Department of Health (DH) (2014). The NHS Outcomes Framework 2015/16. Available online at: www.dh.gov.uk/publications (Accessed 02.09.2015)

Department of Work and Pensions (2011). Health at work - an independent review of sickness absence. Cmnd 8205. London: Her Majesty's Stationary Office.

Department of Work and Pensions (2013). Fitness for work: the government response to 'Health at work- an independent review of sickness absence'. Cmnd 8476. London: Her Majesty's Stationary Office.

Feng J, Chang Y, Chang H, Erdley W, Lin C, Chang Y. (2013) Systematic review of effectiveness of situated elearning on medical and nursing education. Worldviews on Evidence-Based Nursing 10(3): 174-183.

Government Digital Strategy (2013). Available online at: https://www.gov.uk/government/publications/government-digital-strategy. (Accessed 02.09.2015) Health and Safety Executive (HSE) (2010). Stress. Available online at: www.hse.gov.uk/stress/index.htm (Accessed 02.09.2015)

Health and Safety Executive (HSE) (2013). A guide to health and safety regulation in Great Britain. HSE49. Available online at: www.hse.gov.uk (Accessed 02.09.15)

Hegney D, Tuckett A, Parker D, Robert E (2010). Access to and support for continuing professional education amongst Queensland nurses: 2004 and 2007. Nurse Education Today, 27 (6) 602-9. 
Holloway, I. and Wheeler, S. (2013). Qualitative Research in Nursing and Health Care. New York: John Wiley \& Sons Inc.

Horton, W. (2006). E-learning by design. New York: John Wiley \& Sons Inc.

Jeffries, P. (2001). Computer versus lecture: a comparison of two methods of teaching oral medication administration in a nursing skills laboratory. Journal of Nursing Education 40: 323-329.

Joint information systems committee (2004). Effective Practice with e-Learning A good practice guide in designing for learning. London: Higher Education Funding Council for England.

Keefe, G. and Wharrad, H. (2012). Using e-learning to enhance nursing students' pain management education. Nurse Education Today 32(8): 66-72.

Lahti, M., Hatonen, H. and Valimaki, M. (2014). Impact of e-learning on nurses' and student nurses knowledge, skills, and satisfaction: A systematic review and meta-analysis. International journal of nursing studies 51(1): 136-49.

Lotrecchiano, G.; McDonald, P.; Lyons, L.; Long, T.; Zajicek-Farber, M. (2013). Blended Learning: Strengths, Challenges, and Lessons Learned in an Interprofessional Training Program. Maternal \& Child Health Journal, 17 (9): 1725-34.

Lymn, J., Bath-Hextall, F. and Wharrad, H. (2008) Pharmacology education for nurse prescribing students - a lesson in reusable learning objects. BMC Nursing 7(2): 1-11.

McVeigh H. (2009). Factors influencing the utilisation of e-learning in post-registration nursing students. Nurse Education Today 29(1): 91-99..

Mo, P., Blake, H. and Batt, M. (2011). Getting healthcare staff more active: The mediating role of self-efficacy. British Journal of Health Psychology 16(4): 690-706.

Mooney, B., Timmins, F., Byrne, G, and Corroon, A. (2011) Nursing students' attitudes to health promotion to: Implications for teaching practice. Nurse Education Today 31(8): 841-848. 
Mulgan, G. (2010). Influencing Public behaviour to Improve Health and Wellbeing An independent report. Available online at: www.dh.gov.uk/publications (Accessed: 17.09.2015).

Musich, S., Schubiner, H., and McDonald, T. (2009). Keeping healthy workers healthy: creating a culture of health. In N. P. Pronk (Ed.), ACSM's worksite health handbook: a guide to building healthy and productive companies (2nd ed). Champaign, IL (USA): Human Kinetics Publishers.

NHS (2014), Five Year Forward View. Available online at: http://www.england.nhs.uk/wpcontent/uploads/2014/10/5yfv-web.pdf. (Accessed 02.09.2015).

National Information Board (NIB) (2014). Personalised Health and Care 2020 Using Data and Technology to Transform Outcomes for Patients and Citizens A Framework for Action. London: HM Government.

National Institute for Health and Clinical Excellence (NICE) (2008). Workplace health promotion: How to encourage employees to be physically active. Available online at: www.nice.org.uk (Accessed: 04.09.2015).

Office for National Statistics (ONS) (2013). Leading Causes of Death in England and Wales, 2012. Available online at: www.ons.gov.uk (Accessed: 02.09.15).

Office for National Statistics (ONS) (2014). Full Report: Sickness Absence in the Labour Market, February 2014 Available online at: www.ons.gov.uk (Accessed: 02.09.15).

Ousey K, Roberts D. (2012) Improving access to CPD for nurses: the uptake of online provision. British Journal of Community Nursing, $17 ; 12$.

The Point of Care Foundation (2014). Staff Care: how to engage staff in the NHS and why it matters. London: The Point of Care Foundation.

Polit, D. and Tatano-Beck, C. (2008). Nursing Research: Generating and Assessing Evidence for Nursing Practice. Philadelphia: Lippincott Williams and Wilkins.

Polit, D. and Tatano-Beck, C. (2013). Essentials of Nursing Research: Appraising Evidence for Nursing Practice ( $7^{\text {th }}$ Ed). Philadelphia: Lippincott Williams and Wilkins. 
Public Health England (PHE) (2014) Global Health Strategy 2014 to 2019. Available online at:

www.gov.uk/government (Accessed 02.09.2015).

Shareck, M., Frohlich, K. and Poland, B. (2013) Reducing social inequities in health through settings-related interventions -- a conceptual framework. Global Health Promotion 20(2): 39-52.

Smyth, S., Houghton, C., Cooney, A., Casey, D. (2012) Students' experiences of blended learning across a range of postgraduate programmes. Nursing Education Today 32 (4) pp.464-468

Ward R, Moule P, Lockyer L. (2009). Adoption of Web 2.0 Technologies in Education for Health Professionals in the UK: Where Are We and Why? Electronic Journal of e-Learning 7 (2): 165-72.

West, M., Dawson, J., Admasachew, L. and Topakas, A. (2011). NHS Staff Management and Health Service Quality Results from the NHS Staff Survey and Related Data. London: DH.

Wharrad, H., Sanderson-Mann, J., McCandless, F. and Riley, F. (2012). Using reusable learning objects to raise awareness and disseminate research findings about the impact of dyslexia on placement-based learning. Diversity \& Equality in Health \& Care 9(2): 141-149.

Windle, R. Laverty, H., Herman, L., Hallawell, B. and Wharrad, H. (2010). Nursing students can teach their peers by developing e-learning resources. Learning Disability Practice 13(9): 26-29.

World Health Organisation and World Economic Forum (WHO/WEF) (2008). Preventing noncommunicable diseases in the workplace through diet and physical activity - WHO/World Economic Forum Report of a Joint Event. Geneva, Switzerland: World Health Organization.

World Health Organisation (WHO) (2011). Global status report on noncommunicable diseases 2010. Geneva: WHO.

World Health Organisation (WHO) (2014). World Health Statistics 2014. Geneva: WHO. 
